\title{
Modeling of associated tW single top production including the interference with $\mathrm{t} \overline{\mathrm{t}}$ diagrams
}

\author{
Edward Boos ${ }^{1,2, \star}$, Viacheslav Bunichev ${ }^{1, \star \star}$, Lev Dudko $^{1, \star \star \star}$, Igor Myagkov ${ }^{1, \star \star \star \star}$, and Maxim \\ Perfilov ${ }^{1, \dagger}$ \\ ${ }^{1}$ Lomonosov Moscow State University, Skobeltsyn Institute of Nuclear Physics (SINP MSU), 1(2), Leninskie \\ gory, GSP-1, Moscow 119991, Russian Federation \\ ${ }^{2}$ Faculty of Physics M.V.Lomonosov Moscow State University Leninskie Gory, Moscow 119991, Russian \\ Federation
}

\begin{abstract}
The associated tW single top production channel is one of the main mechanism of the electroweak production of top quarks at the LHC. Correct simulation of the SM prediction for this process is one of the important tasks in the SM measurements and BSM searches. One of the difficulties to simulate it correctly is a significant contribution of the interference terms with the pair top quark production. In this article authors compare most popular simulation schemes for $\mathrm{tW}$ production with simulation of the complete set of diagrams and propose new scheme to simulate interference terms without negative weights of the events.
\end{abstract}

\section{Introduction}

Electroweak top quark production is one of the most promising topic to search for New Physics. These are unique processes to measure some of the SM parameters. There are three different production channels. They are classified using the topology of diagrams which include W-boson [1] as $\mathrm{t}$-channel, s-channel and associated $\mathrm{tW}$ production. The associative tW channel becomes important at the LHC and requires proper simulation. The correct simulation of the tW process is difficult due to the well known problem [2]-[8] that at NLO level tW diagrams interfere with pair top quark production diagrams. The top quark pair production has more than ten times larger cross section than the associated $\mathrm{tW}$ production and interference is an important part in case of the precise measurements of tW process. There are different approaches to simulate associated tW production with different advantages and disadvantages. It is also possible to simulate complete set of tree level diagrams including pair top quark production and interference terms. In the next sections we discuss different popular approaches, simulation of the complete set of Feynman diagrams and new scheme proposed

\footnotetext{
${ }^{\star}$ e-mail: boos@theory.sinp.msu.ru

$\star \star$ e-mail: bunichev@ theory.sinp.msu.ru

$\star \star \star$ e-mail: dudko@sinp.msu.ru

$\star \star \star \star$ e-mail: imyagkov@cern.ch

†e-mail: maxim.perfilov@cern.ch
} 


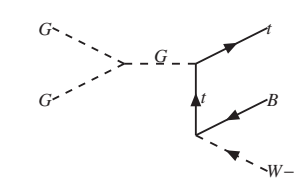

diagr.1

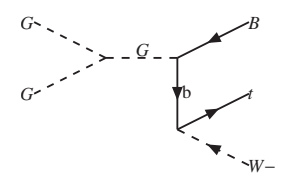

diagr.4

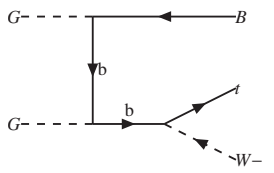

diagr.7

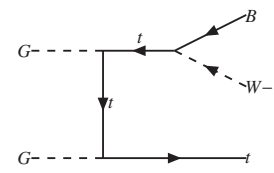

diagr.2

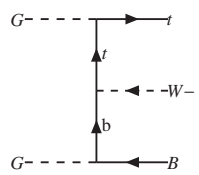

diagr.5

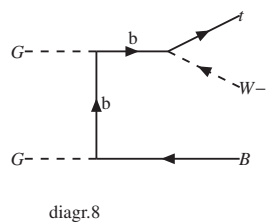

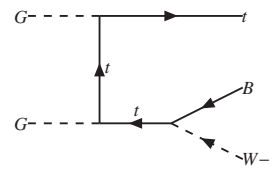

diagr.3

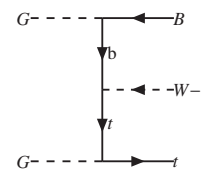

diagr.6

Figure 1: Diagrams for the process $g g \rightarrow t \bar{b} W^{-}$.

in this article which we call "contact interactions" (CI) scheme. The CI scheme solves number of simulation problems. These schemes were implemented in CompHEP $[9,10]$ package and the necessary simulations have been performed. The results are presented in the next sections.

\section{Modeling approaches}

The main LO contribution to the tW process comes from subprocesses with b-quark in the initial state. At NLO level this contribution yields from gluon collisions and leads to the process $g g \rightarrow$ $t W b$ which includes 8 diagrams. The corresponding diagrams are presented in Fig. 1. Simulation of all these diagrams is the most precise approach to model this process. However, part of these diagrams correspond to the pair top quark production with significantly lager cross section than the other diagrams with single top quark production. It is possible to distinguish double resonant diagrams with pair top quark production and single resonant diagrams with single top quark production. In additional, there is significant interference between double and single resonant diagrams. Accurate simulation of the associated tW electroweak single top quark production have to take into account not only single resonant diagrams, but also the negative interference terms with pair top quark production. Different approximate schemes have been developed to properly model tW channel of single top quark production.

Diagram Removal. The most simple recipe is to remove all Feynman diagrams with pair top quark production, diagrams 1-3 in Fig 1. Such approach is called "Diagram Removal" (DR) scheme $[2,3,7]$. In this approach the interference of single resonant diagrams (diagrams 4-8 in Fig. 1) and double resonant diagrams (diagrams 1-3 in Fig. 1) are not taken into account.

Diagram Subtraction. More sophisticated approach called Diagram Subtraction $[3,7]$ takes into account interference terms. This method implies removing only squared double resonant diagrams 

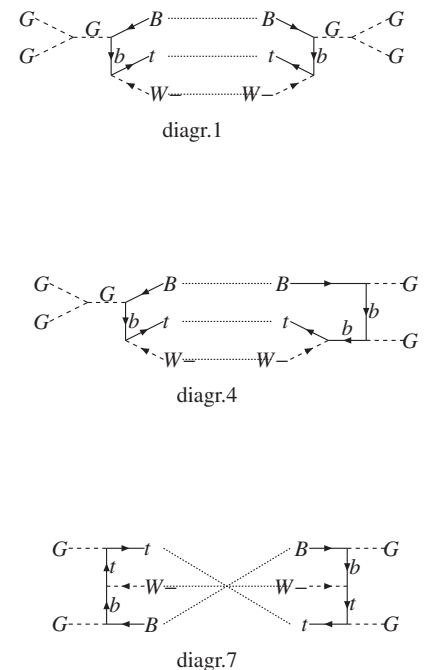

diagr.7

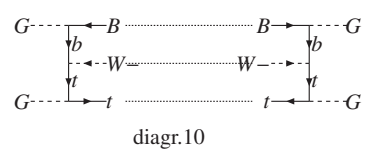

diagr. 10

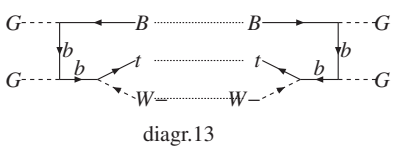

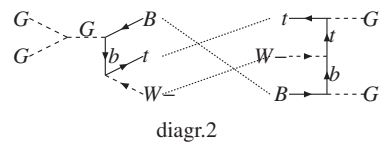
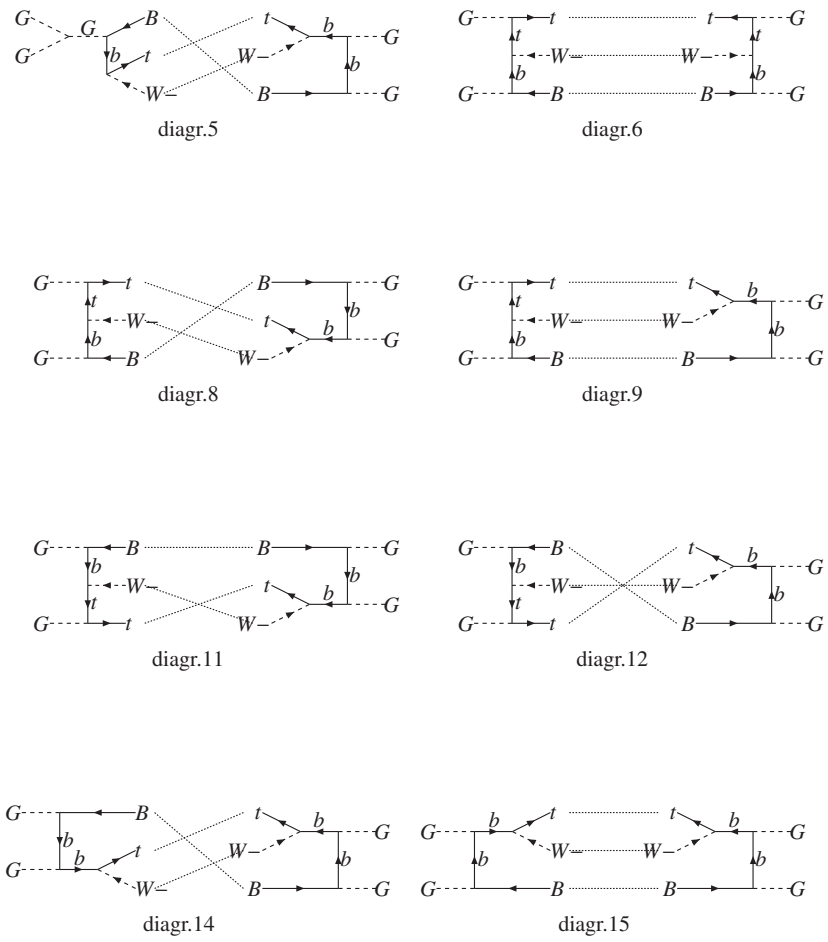

Figure 2: Squared single resonant diagrams for the process $g g \rightarrow t \bar{b} W^{-}$.

during the modeling. The remaining diagrams are shown in Fig. 2 for the single resonant contribution and in Fig. 3 for the interference between single and double resonant contributions. This approach allows to retain all of anticipated single top quark production properties in the $W t b$ vertex. On the other hand this subset of diagrams also introduces extra singularities from an uncoupled top quark decaying into $\mathrm{W}$ boson and $\mathrm{b}$ quark in either part of squared diagrams. This effect can be clearly observed in a distribution of invariant mass of system of $\mathrm{W}$ boson and $\mathrm{b}$ quark, and leads to negative weights of the significant part of the simulated events.

Contact interactions scheme. An improvement can be done to previous scheme in order to get rid of described singularities. These singularities can be excluded entirely by replacing top quark decaying into $\mathrm{W}$ boson and $\mathrm{b}$ quark by a single contact interaction in the double resonant part of the squared interference diagrams in Fig. 3. In this case the distribution of invariant mass of $\mathrm{W}$ and $\mathrm{b}$ turns up to be single-top-like while keeping all interference in place without negative contribution.

Full scheme. Modeling the complete set of diagrams is the most precise approach. For the SM measurements this is not very interesting approach since for the most of the SM measurements one 

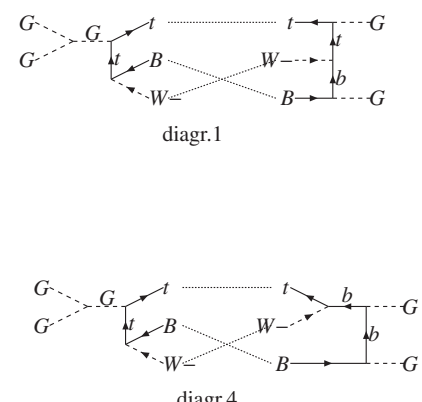

diagr.4
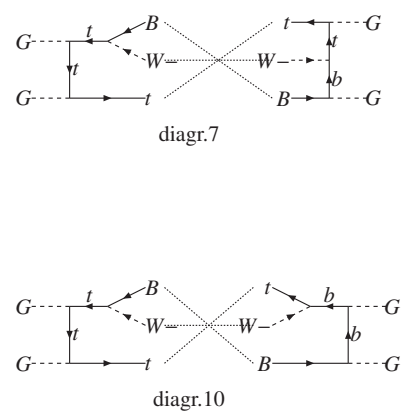

diagr. 10

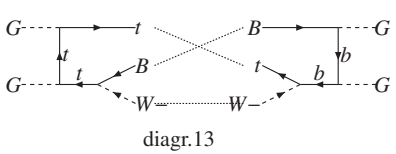

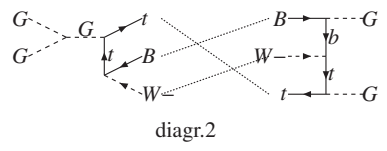
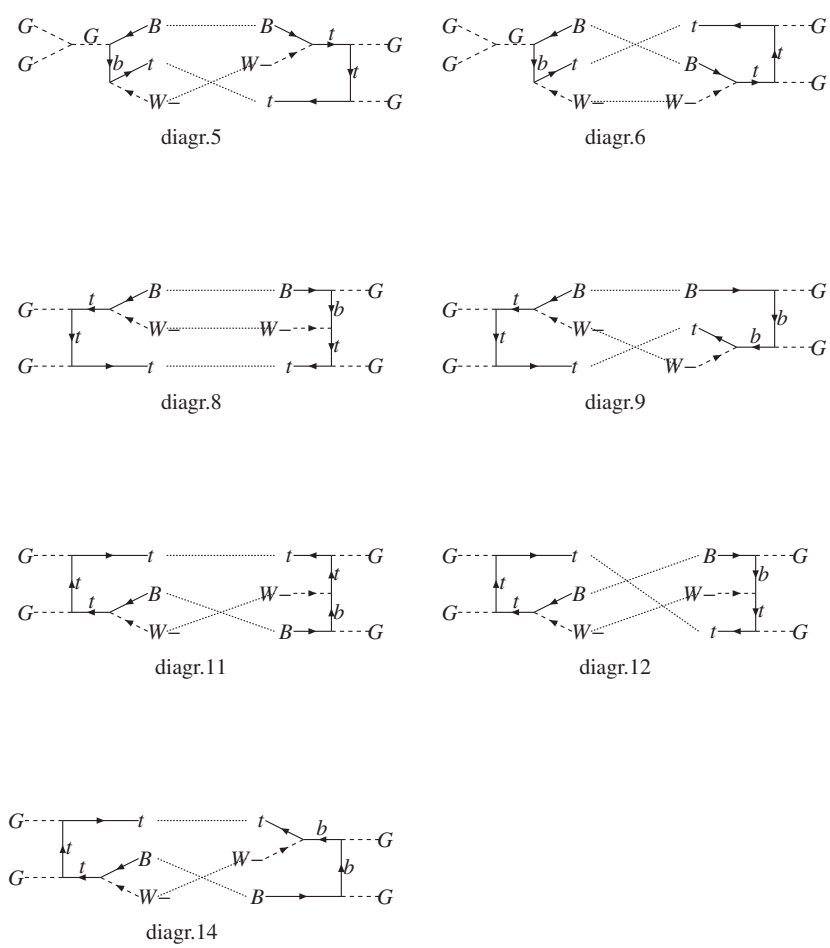
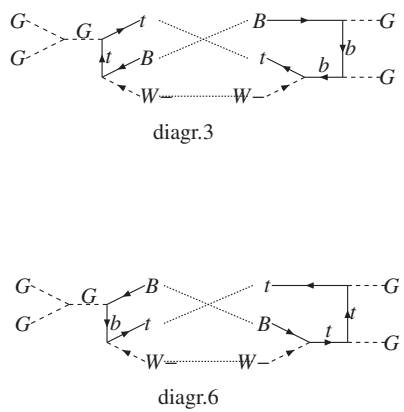

diagr.6

Figure 3: Squared interference diagrams of single and double resonant contributions for the process $g g \rightarrow t \bar{b} W^{-}$.

needs to separate top quark single electroweak production mode and pair production mode in strong interactions. In the full modeling scheme, which will be referenced as a "Full scheme", different terms contribute in total cross section. These are (as shown in Fig. 1) resonant paired production diagrams, resonant single top production diagrams, and their interfere terms. To retain all single top-sensitive interactions, interference terms need to be studied as well as resonant single top terms.

\section{Comparison}

In the previous section we discuss different schemes to simulate tW with different contributions. In this section we compare the common kinematic distributions achieved in different schemes. Figures 417 give a vision of different impacts of particular schemes. Since the cross sections are significantly different for double resonant and single resonant contributions each curve is normilised to its integral (total cross section for this contribution) for the Figs. 4-15. The curves for DR, DS, contact interactions approaches, full scheme and pair top quark production are shown on the plots. The curve for 
the interference term between paired and single production processes is shown on the plots as well. The transverse momenta and pseudorapidity of the t, W, b are shown in Figs. 4-6 and Figs. 8-10. The vector sum of $\mathrm{W}$ boson and b-quark transverse momenta and its pseudorapidity is shown in Fig. 7 and Fig. 11. The significant difference between simulation approaches is visible in the shapes of b-quark and $W b$ system distributions. The angular distributions are show in Figs. 12-15. The cosine of angle between $\mathrm{W}$ and $\mathrm{t}$ momenta in the laboratory rest frame and in $\mathrm{Wb}$ rest frame are shown in Figs. 12 and Figs. 13 correspondingly. The cosine of angle between $\mathrm{t}$ and $\mathrm{b}$ quarks momenta are shown in Figs. 14 and Figs. 15 correspondingly. Angular distributions demonstrate significant difference of DS and CI schemes from other simulation approaches. This is reasonable, since the DS and DI schemes takes into account the interference terms. The differential cross section of invariant mass of $\mathrm{W}$ boson and b-quark is shown in Fig. 16. The distribution of invariant mass of $\mathrm{W}$ boson and b-quark for the interference term is presented in Fig. 17.

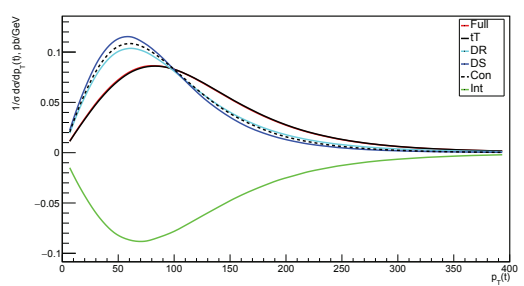

Figure 4: Transverse momentum of top quark

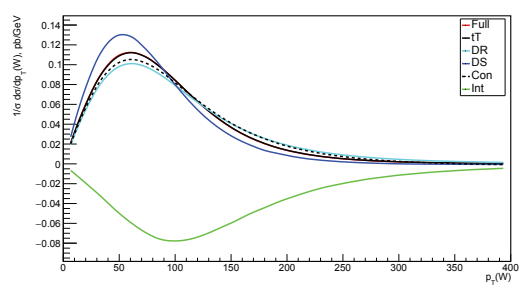

Figure 6: Transverse momentum of $\mathrm{W}$ boson

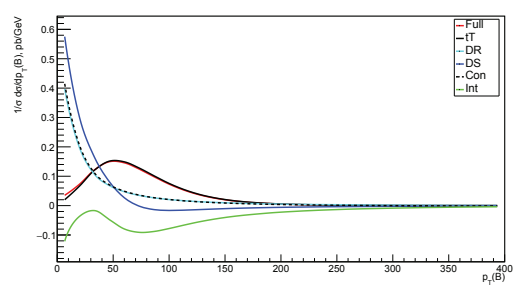

Figure 5: Transverse momentum of $b$ quark.

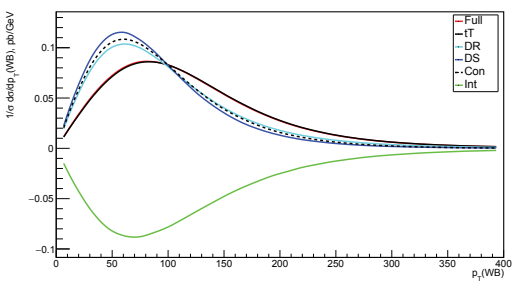

Figure 7: Transverse momentum of system of $\mathrm{W}$ boson and $\mathrm{b}$ quark.

\section{Kinematic separation}

Kinematic separation of double and single resonant contributions is also possible [2, 3]. Such approach takes into account interference terms, it is gauge invariant and avoids negative weights of the events. In this section we compare simulation in different approximate schemes with the simulation of complete set of diagrams. For the calculations we exclude phase space region $145<M_{W b}<200$ $\mathrm{GeV}$ which corresponds to the second top quark pole. In this way the cross sections for all schemes are comparable. The correct simulation should correspond to single resonant diagrams with interference term, and off-shell contribution for the double resonant diagrams. Simulation of the complete set 


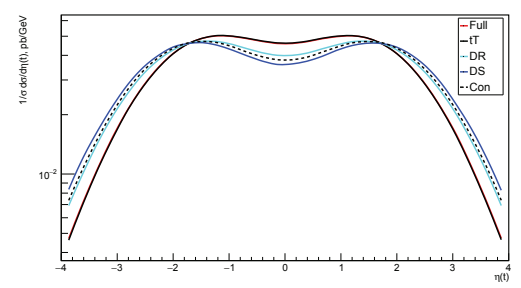

Figure 8: Pseudorapidity of top quark.

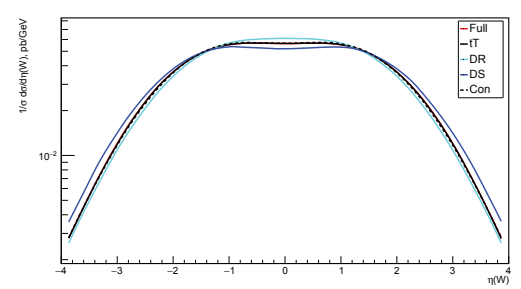

Figure 10: Pseudorapidity of $\mathrm{W}$ boson. Figure 11: Pseudorapidity of system of

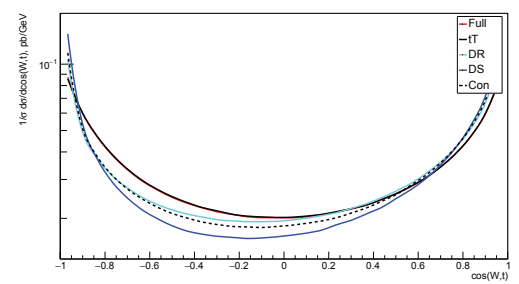

Figure 12: Cosine between $\mathrm{W}$ boson and top quark.

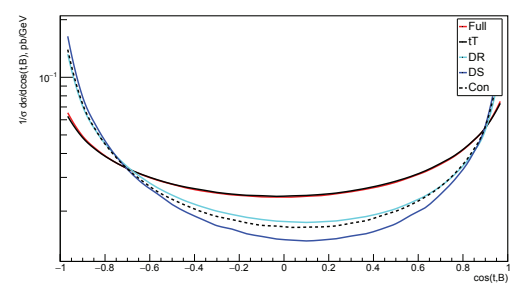

Figure 14: Cosine between top quark and $b$ quark. $\mathrm{W}$ boson and $\mathrm{b}$ quark.

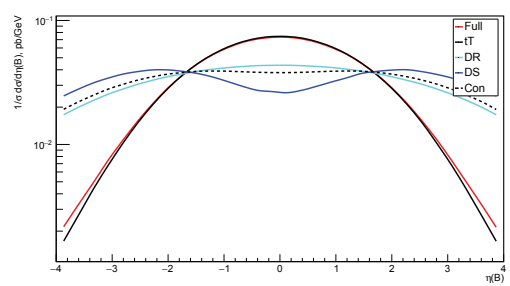

Figure 9: Pseudorapidity of b quark.
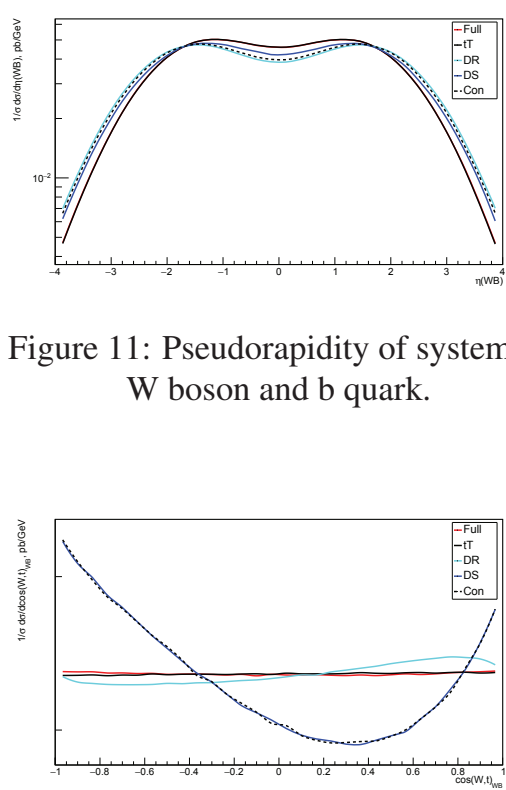

Figure 13: Cosine between $\mathrm{W}$ boson and top quark in rest frame of $\mathrm{W}$ and $\mathrm{b}$.

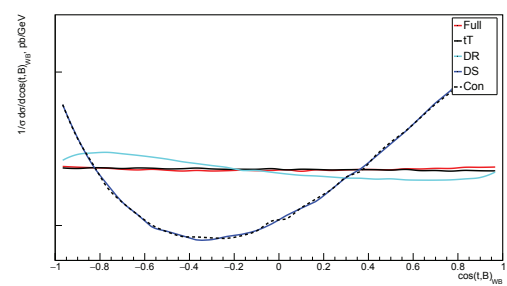

Figure 15: Cosine between top quark and $\mathrm{b}$ quark in rest frame of $\mathrm{W}$ and $\mathrm{b}$. 


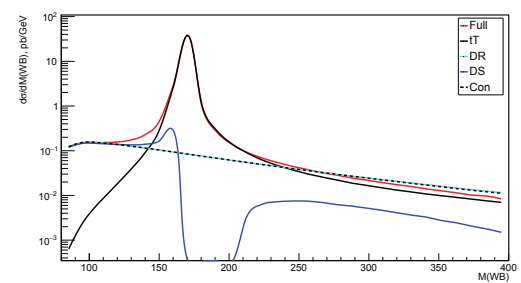

Figure 16: Invariant mass of $W$ boson and $b$ quark, no interference term.

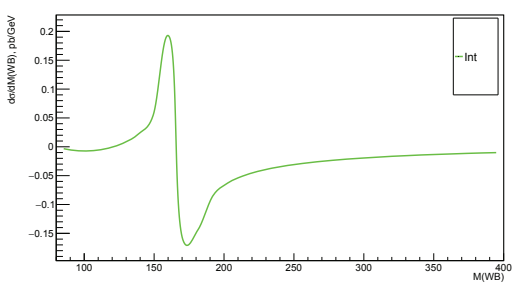

Figure 17: Invariant mass of $W$ boson and $b$ quark, only interference term.

of diagrams ("Full scheme") is most accurate way to model this process in this phase space region. Kinematic distributions in Figs. 18-27 demonstrate the difference between DR, DS, CI schemes and full scheme. The contribution of the negative interference is also shown as well as pure double resonant contribution. DR scheme should reproduce only single resonant contribution. DS scheme have to take into account single resonant contribution and negative interference. CI scheme is designed to reproduce kinematics of the single resonant contribution with interference term, but without negative events. Double resonant contribution reproduces only pair top quark production. Full scheme reproduces all contributions, single resonant, double resonant and their interference.

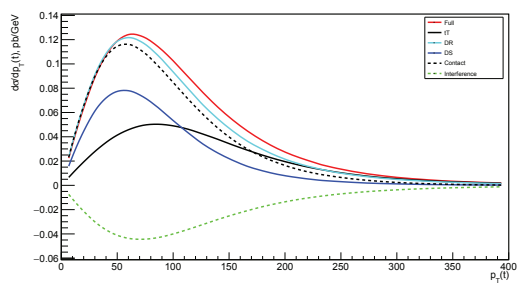

Figure 18: Transverse momentum of top quark with excluded region $145<M_{W b}<200 \mathrm{GeV}$

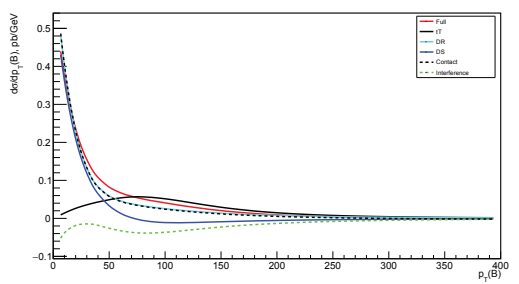

Figure 19: Transverse momentum of $b$ quark with excluded region $145<M_{W b}<200 \mathrm{GeV}$.

\section{Conclusion}

Presented techniques allow different approaches to simulate tWb production and may be useful for different particular analyses. However, only modeling of the complete set of Feynman diagrams retains correct distributions of kinematic variables. The "Diagram Removal" scheme discards all interference terms which are significant part of single top quark production processes. The "Diagram Subtraction" scheme provides best differentiation in angular distributions and could be useful for separation of single top quark production, but introduces negative weights for the significant part of the events and barely can be used in the analysis. The improved "Contact interaction" scheme does not have events with negative weights and shows little difference from "Diagram subtraction" scheme for the angular distributions, thus could be used for effective simulation and separation of single top quark production process. 


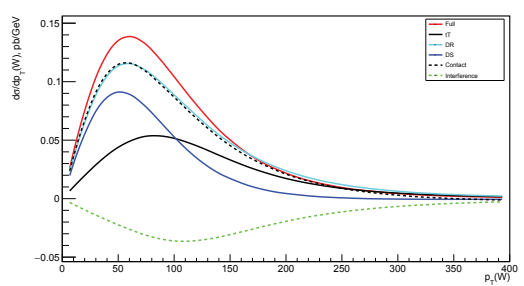

Figure 20: Transverse momentum of $\mathrm{W}$ boson with excluded region $145<M_{W b}<200 \mathrm{GeV}$

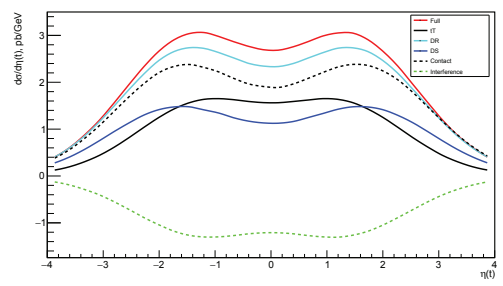

Figure 22: Pseudorapidity of top quark with excluded region $145<M_{W b}<200$

$\mathrm{GeV}$.

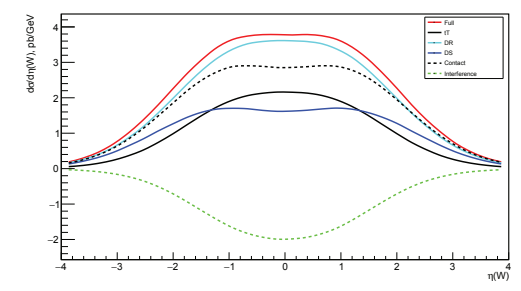

Figure 24: Pseudorapidity of $W$ boson Figure 25: Pseudorapidity of system of with excluded region $145<M_{W b}<200$ $\mathrm{GeV}$.

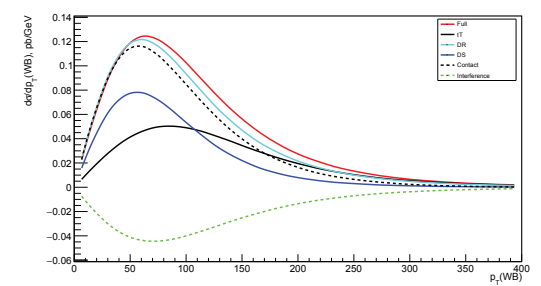

Figure 21: Transverse momentum of system of $\mathrm{W}$ boson and $\mathrm{b}$ quark with excluded region $145<M_{W b}<200 \mathrm{GeV}$.

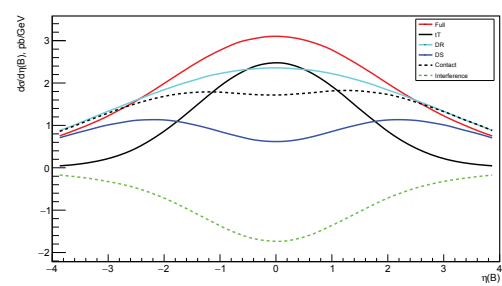

Figure 23: Pseudorapidity of $b$ quark with excluded region $145<M_{W b}<200$

$\mathrm{GeV}$.

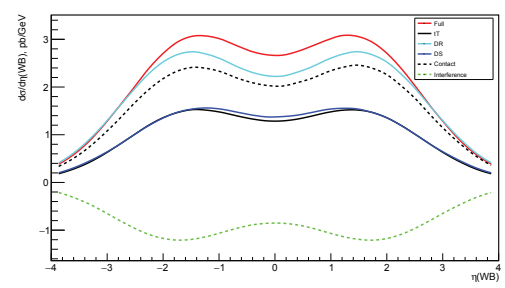

$W$ boson and $b$ quark with excluded region $145<M_{W b}<200 \mathrm{GeV}$.

Kinematic cut based approach, as shown in Section 4 is generally less sensitive to electroweak properties since important part of phase space is discarded and the impact from paired top quark production is not reduced entirely. Nonetheless, such approach takes into account all necessary contributions and gives a more stable result because it uses purely gauge invariant kinematic reduction procedure. Kinematic separation does not have negative weights of the events and can be used in the searches for the deviations from the SM. 


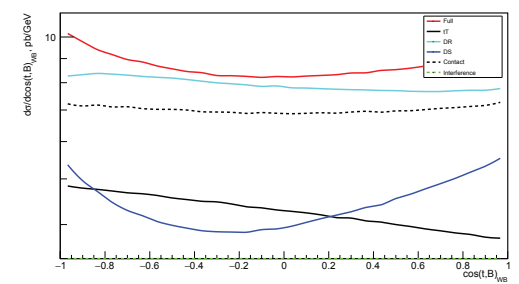

Figure 26: Cosine between top quark and $b$ quark in rest frame of $W$ and $b$ with excluded region $145<M_{W b}<200$ $\mathrm{GeV}$.

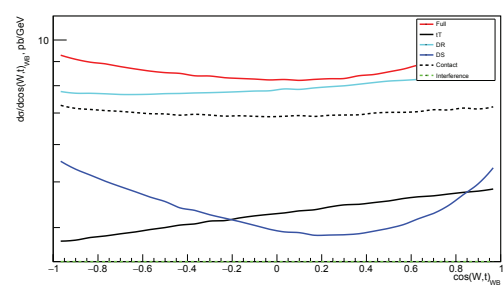

Figure 27: Cosine between $W$ boson and top quark in rest frame of $W$ and $b$ with excluded region $145<M_{W b}<200$

$\mathrm{GeV}$.

\section{Acknowledgments}

The work was supported by grant 16-12-10280 of Russian Science Foundation.

\section{References}

[1] S. S. D. Willenbrock and D. A. Dicus, Phys. Rev. D 34, 155 (1986). doi:10.1103/PhysRevD.34.155

[2] A. S. Belyaev, E. E. Boos and L. V. Dudko, Phys. Rev. D 59, 075001 (1999) doi:10.1103/PhysRevD.59.075001 [hep-ph/9806332].

[3] T. M. P. Tait, Phys. Rev. D 61, 034001 (1999) doi:10.1103/PhysRevD.61.034001 [hep$\mathrm{ph} / 9909352]$.

[4] S. Zhu, Phys. Lett. B 524, 283 (2002) Erratum: [Phys. Lett. B 537, 351 (2002)] doi:10.1016/S0370-2693(02)01952-4, 10.1016/S0370-2693(01)01404-6 [hep-ph/0109269].

[5] J. M. Campbell and F. Tramontano, Nucl. Phys. B 726, 109 (2005) doi:10.1016/j.nuclphysb.2005.08.015 [hep-ph/0506289].

[6] B. P. Kersevan and I. Hinchliffe, JHEP 0609, 033 (2006) doi:10.1088/1126-6708/2006/09/033 [hep-ph/0603068].

[7] S. Frixione, E. Laenen, P. Motylinski, B. R. Webber and C. D. White, JHEP 0807, 029 (2008) doi:10.1088/1126-6708/2008/07/029 [arXiv:0805.3067 [hep-ph]].

[8] C. D. White, S. Frixione, E. Laenen and F. Maltoni, JHEP 0911, 074 (2009) doi:10.1088/11266708/2009/11/074 [arXiv:0908.0631 [hep-ph]].

[9] E. Boos et al. [CompHEP Collaboration], Nucl. Instrum. Meth. A 534, 250 (2004) doi:10.1016/j.nima.2004.07.096 [hep-ph/0403113].

[10] E. Boos et al., PoS ACAT 08, 008 (2008) [arXiv:0901.4757 [hep-ph]]. 